\title{
Exploring configurational degrees of freedom in disordered solids
}

Cite as: AIP Conference Proceedings 2040, 020003 (2018); https://doi.org/10.1063/1.5079045 Published Online: 30 November 2018

Pavel A. Korzhavyi, Yunguo Li, Inna L. Soroka, and Claudio M. Lousada

ARTICLES YOU MAY BE INTERESTED IN

Preface: Proceedings of the International Conference of Computational Methods in Sciences and Engineering 2018 (ICCMSE-2018)

AIP Conference Proceedings 2040, 010001 (2018); https://doi.org/10.1063/1.5079040

Preface of "The 4th Computational Chemistry (CC) Symposium"

AIP Conference Proceedings 2040, 020001 (2018); https://doi.org/10.1063/1.5079043

A theoretical study of hydrogen anion transport in $\mathrm{BaTiO}_{3}$ perovskite

AIP Conference Proceedings 2040, 020002 (2018); https://doi.org/10.1063/1.5079044

\section{Conference Proceedings}

Get $30 \%$ off all print proceedings!

Enter Promotion Code P/DF=30 at checkout

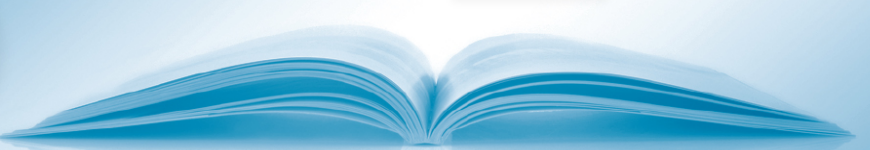




\title{
Exploring Configurational Degrees of Freedom in Disordered Solids
}

\author{
Pavel A. Korzhavyi ${ }^{1,2, \text { a) }}$, Yunguo Li ${ }^{1,3}$, Inna L. Soroka ${ }^{4}$, and Claudio M. Lousada ${ }^{1}$ \\ ${ }^{1}$ Department of Materials Science and Engineering, KTH - Royal Institute of Technology, Stockholm, Sweden \\ ${ }^{2}$ Materials Modeling and Development Laboratory, National University of Science and Technology "MISiS", \\ Moscow, Russia \\ ${ }^{3}$ Faculty of Mathematical and Physical Sciences, University College London, United Kingdom \\ ${ }^{4}$ Applied Physical Chemistry, School of Chemical Science and Engineering, KTH - Royal Institute of Technology, \\ Stockholm, Sweden \\ ${ }^{a)}$ Corresponding author: pavelk@kth.se
}

\begin{abstract}
Recent computational modeling studies of configurational degrees of freedom in oxide-hydroxides of aluminum and copper are reviewed. Density functional theory calculations are employed to investigate the effect of hydrogen on the structural stability of gamma-alumina and to explore the configurational space of cuprous hydroxide $\mathrm{CuOH}$. Free energy modeling, taking into account configurational and vibrational degrees of freedom, shows that the studied hydrogenated oxides of aluminum and copper are metastable compounds, whereas the respective oxides are thermodynamically stable phases. Selected structural and optical properties of the studied compounds, evaluated using a hybrid functional approach, are reported and compared with available experimental data.
\end{abstract}

\section{INTRODUCTION}

The crystal structures of many oxides and hydroxides exhibit configurational disorder which makes important contributions to the physical and chemical properties of these compounds. In our recent studies [1-5] we employed ab initio calculations based on density functional theory (DFT) to study the relationships between the structural characteristics of oxide-hydroxides of aluminum and copper and their physical and chemical properties. In particular, we considered different structural models of pure and hydrogen-bearing $\gamma-\mathrm{Al}_{2} \mathrm{O}_{3}$ to investigate the effect of hydrogen on the structural stability of gamma-alumina [2]. Also, the configurational space of cuprous hydroxide $\mathrm{CuOH}$ was systematically explored to identify the most energetically favorable structure and to accurately estimate its thermodynamic properties [4,5]. When estimating the free energy of the competing structural models of gammaalumina and cuprous hydroxide, it is essential to take into account the configurational entropy $[2,4]$. The available configurational space of $\mathrm{CuOH}$ has been systematically explored to identify the energetically favorable configurations [4]. The origin of structural preference and its relation to the electronic structure of gamma-alumina and cuprous hydroxide are briefly discussed below.

\section{CONFIGURATIONAL DISORDER IN ALUMINAS}

Besides the most stable form of alumina, $\alpha-\mathrm{Al}_{2} \mathrm{O}_{3}$, there are also several transition aluminas that can be obtained by thermal decomposition of aluminum hydroxides or oxyhydroxides. The $\gamma$-alumina phase has been extensively studied mostly due to its applications as a catalyst or catalyst support. Its crystal structure is based on the cubic spinel structure of $\mathrm{MgAl}_{2} \mathrm{O}_{4}$. The cubic unit cell of this magnesium aluminum spinel comprises $8 \mathrm{Mg}$ cations (at $8 a$ sites), $16 \mathrm{Al}$ cations (at $16 d$ sites), and 32 close-packed $\mathrm{O}$ anions (at $32 e$ sites). The cation sites in $\gamma$-alumina are not fully occupied: in order to meet the $\mathrm{Al}_{2} \mathrm{O}_{3}$ stoichiometry, $2{ }_{2}^{2} 3 \mathrm{Al}$ cation sites per unit cell (containing 32 anions)

International Conference of Computational Methods in Sciences and Engineering 2018 (ICCMSE 2018)

AIP Conf. Proc. 2040, 020003-1-020003-4; https://doi.org/10.1063/1.5079045

Published by AIP Publishing. 978-0-7354-1766-3/\$30.00 
have to be vacant. It is still a matter of debate which cation sites are preferred by the vacancies. These are so-called vacated models of $\gamma$-alumina.

Another type of suggested structures is a hydrogenated or protonated model of $\gamma$-alumina. There the balance between the cations and anions is kept due to the partial filling of cation vacancies by hydrogen cations (protons) to form $\mathrm{OH}^{-}$groups. Such a structural model can formally be described as $\gamma-\mathrm{Al}_{2} \mathrm{O}_{3} \cdot{ }_{1}{ }_{5} \mathrm{H}_{2} \mathrm{O}$, although it is to denote an oxyhydroxide rather than a hydrated oxide. The amount of $\mathrm{Al}$ vacancies in the hydrogenated model is therefore reduced compared to the vacated model of $\gamma$-alumina, but the former model possesses an additional type of configurational disorder related to the position of hydrogen in the structure (proton disorder). It is therefore interesting to compare these two structural models in terms of the maximum configurational entropy they can possess.

Pauling's assumption [6] that all 9 cation sites in the minimal primitive cell $\mathrm{Al}_{8} \mathrm{O}_{12}$ of $\gamma$-alumina structure are occupied by $8 \mathrm{Al}^{3-}$ ions completely at random gives the following upper-bound estimate for the configurational entropy $S_{\mathrm{A}}$ (per mole of $\mathrm{Al}_{2} \mathrm{O}_{3}$ molecules):

$$
S_{\mathrm{A}}^{\max }=-\frac{9}{4} R\left[\frac{1}{9} \ln \frac{1}{9}+\frac{8}{9} \ln \frac{8}{9}\right]=\frac{1}{4} R \ln \left(\frac{9^{9}}{8^{8}}\right)=\frac{3}{2} R \ln \left(\frac{27}{16}\right) \cong 0.7849 \cdot R=6.53 \mathrm{~J} \cdot \mathrm{mol}^{-1} \cdot \mathrm{K}^{-1} .
$$

Here $R$ is the gas constant. A charge-balanced formula of hydrogenated spinel, $\mathrm{Al}_{5} \mathrm{HO}_{8}$, corresponds to one protonated vacancy per 6 cation sites ( 2 tetrahedral and 4 octahedral). The proton inside such a vacancy binds to one of the neighboring $\mathrm{O}$ anions to form an $\mathrm{OH}^{-}$group. Assuming a completely random substitution of any vacant cation site by hydrogen (and also that $\mathrm{OH}^{-}$formation is completely uncorrelated), one arrives at the following upper-bound estimate of configurational entropy (per formula $\mathrm{Al}_{2} \mathrm{O}_{3} \cdot{ }_{1} / \mathrm{H}_{2} \mathrm{O}$ ):

$$
S_{\mathrm{H}}^{\max }=\frac{2}{5} R\left[\ln \frac{6^{6}}{5^{5}}+\left(\frac{2}{6} \ln 4+\frac{4}{6} \ln 6\right)\right] \cong 1.7440 \cdot R=14.500 \mathrm{~J} \cdot \mathrm{mol}^{-1} \cdot \mathrm{K}^{-1}
$$

where the largest part of the entropy, $1.0813 \cdot R$ or $8.991 \mathrm{~kJ} \cdot \mathrm{mol}^{-1} \cdot \mathrm{K}^{-1}$, is due to the randomness of vacancy distribution, while the remaining part, $0.6626 \cdot R$ or $5.509 \mathrm{~J} \cdot \mathrm{mol}^{-1} \cdot \mathrm{K}^{-1}$, is due to the proton disorder.

\section{CONFIGURATIONAL DISORDER IN CUPROUS OXIDE AND HYDROXIDE}

Cuprous oxide $\mathrm{Cu}_{2} \mathrm{O}$ is probably the best studied compound of copper. It has the cuprite crystal structure, which can be viewed as consisting of two interpenetrating anti-cristobalite lattices. The $\mathrm{O}^{2-}$ anion sublattice is bodycentered cubic (bcc) and splits into two diamond lattices when the ionic coordination is considered: each $\mathrm{O}^{2-}$ anion is tetrahedrally coordinated by four $\mathrm{Cu}^{+}$cations, while each $\mathrm{Cu}+$ cation is linearly coordinated by two anions. All the $\mathrm{Cu}^{+}$ions taken together constitute a face-centered cubic (fcc) cation sublattice in $\mathrm{Cu}_{2} \mathrm{O}$. Using a ball-and-stick model, it is easy to notice that the two identical anti-cristobalite $\mathrm{Cu}_{2} \mathrm{O}$ lattices composing the cuprite structure are not connected to each other by primary $\mathrm{Cu}-\mathrm{O}$ chemical bonds, so that the structure is held together via many-body and secondary (dispersive) interactions.

Very little experimental information is available about the structure and stability of a bulk form of cuprous hydroxide $\mathrm{CuOH}$. In this connection, $\mathrm{CuOH}$ was recently studied theoretically and experimentally [1,3-5]. A structural model of $\mathrm{CuOH}$ was proposed where this compound is viewed as a result of cation substitution of $\mathrm{Cu}^{+}$by $\mathrm{H}^{+}$. The structure resulting from such substitution is intermediate between those of cuprite and ice-IV. Therefore, this structural model of $\mathrm{CuOH}$ was termed "cuprice" [1]. Even if Pauling's coordination rules for a hydroxide are strictly obeyed, there is still some freedom how the $\mathrm{Cu}$ and $\mathrm{H}$ cations can be distributed in $\mathrm{CuOH}$. Using the same counting procedure as that proposed by Pauling [6], one can estimate the maximum entropy of $\mathrm{CuOH}$ cuprice as

$$
S_{\mathrm{CuOH}}^{\max }=R \ln \left(\frac{3}{\sqrt{2}}\right)=6.25 \mathrm{~J} \cdot \mathrm{mol}^{-1} \cdot \mathrm{K}^{-1} \text {. }
$$


TABLE 1. Calculated properties for different structural models of gamma-alumina, cuprous oxide, and cuprous hydroxide in comparison with available experimental data. Equilibrium volume is expressed per formula unit (f.u.) of every substance.

\begin{tabular}{clcc}
\hline Phase & Model, method & Volume $\left(\AA^{\mathbf{3}} / \mathbf{f} . \mathbf{u}.\right)$ & Band gap (eV) \\
\hline$\gamma-\mathrm{Al}_{2} \mathrm{O}_{3}$ & Vacant $\mathrm{Al}$ sites, theory & 45.89 & 6.0 \\
& $\gamma-\mathrm{Al}_{2} \mathrm{O}_{3} \cdot{ }^{1} /{ }_{5} \mathrm{H}_{2} \mathrm{O}$, theory & 45.99 & 6.4 \\
& Experiment & $46.42-46.98^{\mathrm{a}}$ & $2.5-8.7^{\mathrm{b}}$ \\
$\mathrm{Cu}$ & 39.75 & 2.2 \\
& Theory & $38.89^{\mathrm{c}}$ & $2.0-2.2^{\mathrm{d}}$ \\
$\mathrm{CuOH}$ & Experiment & 31.61 & 2.7 \\
& Ferroelectric, theory & 33.73 & 3.0 \\
\hline
\end{tabular}

${ }^{\mathrm{a}}$ Ref. [11] $\quad{ }^{\mathrm{b}}$ Ref. [12] $\quad{ }^{\mathrm{c}}$ Ref. [13] $\quad{ }^{\mathrm{d}}$ Ref. [14]

\section{STRUCTURE, STABILITY, AND OTHER PROPERTIES OF STUDIED COMPOUNDS}

Electronic structure, total energy, and related properties of hydrogen-free and hydrogenated alumina and cuprous oxide were calculated in Refs. [2,5] using the Vienna ab-initio simulation package (VASP) [7, 8] and the PBE0 hybrid functional $[9,10]$. The complete free energy modeling, based on the calculated total energies and phonon spectra, show that hydrogenated $\gamma$-alumina is less stable than the corresponding mixture of hydrogen-free $\gamma-\mathrm{Al}_{2} \mathrm{O}_{3}$ and $\gamma-\mathrm{AlOOH}$ (Boehmite) in the temperature range considered (below $1000 \mathrm{~K}$ ), in spite of the higher configurational entropy of hydrogenated $\gamma$-alumina [2]. The presence of hydrogen is found to slightly increase the equilibrium volume and the band gap of $\gamma$-alumina (calculated for the chosen supercell models of $\gamma-\mathrm{Al}_{2} \mathrm{O}_{3}$ and $\gamma-\mathrm{Al}_{2} \mathrm{O}_{3} \cdot{ }_{1}{ }_{5} \mathrm{H}_{2} \mathrm{O}$ in the ground state), see Table 1 . The table also shows that the obtained difference does not fully account for the experimentally observed variation of molecular volume and band gap of $\gamma$-alumina, which implies that additional factors (the presence of other defects and impurities in the structure) may influence the properties.

To investigate whether the ordering of cations can affect the structural stability and other properties of $\mathrm{CuOH}$ cuprice, we performed a combinatorial study of all possible (symmetry) cation configurations within a supercell containing $16 \mathrm{CuOH}$ formula units [4]. The total number of enumerated configurations corresponds to an entropy value of $5.89 \mathrm{~J} \cdot \mathrm{mol}^{-1} \cdot \mathrm{K}^{-1}$, which is slightly less than the maximum value of $S_{\mathrm{CuOH}}$ given by Eq. (3). Therefore, the chosen supercell size allowed us to explore a considerable portion of the total configurational space available for $\mathrm{CuOH}$. The lowest-energy structure of $\mathrm{CuOH}$ is calculated out to be antiferroelectrically ordered layered structure described in Ref. [4]. It has a wider bandgap and a smaller equilibrium volume than $\mathrm{Cu}_{2} \mathrm{O}$ (see Table 1 and Refs. $[4,5]$ ), and is lower in energy by some $7.5 \mathrm{~kJ} \cdot \mathrm{mol}^{-1}$ than the ferroelectrically-ordered $\mathrm{CuOH}$ structure investigated previously in Ref. [1]. Using the data obtained, the thermodynamic metastability of $\mathrm{CuOH}$ was demonstrated: even if one combines the energy of the ground-state $\mathrm{CuOH}$ structure with the maximum entropy of cuprice, the free energy of $\mathrm{CuOH}$ is calculated to be lower than that of a mixture of $\mathrm{Cu}_{2} \mathrm{O}$ and $\mathrm{H}_{2} \mathrm{O}$.

In summary, we have investigated the structure and properties of hydrogen-free and hydrogenated forms of $\gamma$ alumina and cuprous oxide. The presence of proton disorder in the hydrogenated compounds gives them considerably higher configurational entropy compared to their hydrogen-free counterparts. Summing up all the free energy contributions together, we find that larger configurational entropy is not enough to make $\gamma-\mathrm{Al}_{2} \mathrm{O}_{3} \cdot{ }_{1}{ }_{5} \mathrm{H}_{2} \mathrm{O}$ and $\mathrm{CuOH}$ truly thermodynamically stable phases.

\section{ACKNOWLEDGMENTS}

This work was funded by the Swedish Nuclear Fuel and Waste Management Company (SKB) and the Swedish Foundation for Strategic Research (SSF, project ALUX). P.A.K. gratefully acknowledges the financial support of the Ministry of Education and Science of the Russian Federation in the framework of the Increase Competitiveness Program of NUST "MISiS" (Grant No. K3-2017-034). The research used resources provided by the Swedish National Infrastructure for Computing (SNIC) at the National Supercomputer Centre (NSC), Linköping, the High Performance Computing Center North (HPC2N), and at the PDC Center for High-performance Computing, Stockholm. 


\section{REFERENCES}

1. P.A. Korzhavyi, I. L. Soroka, E. I. Isaev, C. Lilja, and B. Johansson, Proc. Natl. Acad. Sci. 109, 686-689 (2012).

2. Yunguo Li, C. M. Lousada, and P. A. Korzhavyi, J. Appl.Phys. 115, 203514 (2014).

3. I. L. Soroka, A. Shchukarev, M. Jonsson, N. V. Tarakina, and P. A. Korzhavyi, Dalton Trans. 42, 9585-9594 (2013).

4. Yunguo Li, C. M. Lousada, I. L. Soroka, and P. A. Korzhavy, Inorg. Chem. 54, 8969-8977 (2015).

5. Yunguo Li and P. A. Korzhavyi, Dalton Trans. 46, 529-538 (2017).

6. L. Pauling, J. Am. Chem. Soc. 57, 2680-2684 (1935).

7. G. Kresse and J. Furthmüller, Comput. Mater. Sci. 6, 15-50 (1996).

8. G. Kresse and J. Furthmüller, Phys. Rev. B: Condens. Matter 54, 11169 (1996).

9. J. P. Perdew, K. Burke and M. Ernzerhof, Phys. Rev. Lett. 77, 3865-3868 (1996).

10. C. Adamo and V. Barone, J. Chem. Phys. 110, 6158-6170 (1999).

11. R.-S. Zhou and R. L. Snyder, Acta Crystallogr., Sect. B 47, 617 (1991).

12. B. Ealet, M. H. Elyakhloufi, E. Gillet, and M. Ricci, Thin Solid Films 250, 92-100 (1994).

13. A. F. Wells, Structural inorganic chemistry. 5th ed. (Oxford: Clarendon, 1984).

14. S. N. Kale, S. B. Ogale, S. R. Shinde, M. Sahasrabuddhe, V. N. Kulkarni, R. L. Greene and T. Venkatesan, Appl. Phys. Lett. 82, 2100-2102 (2003). 